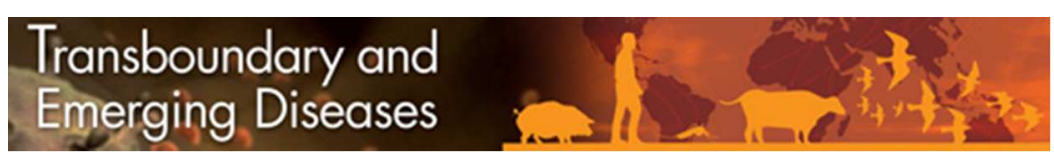

\title{
Genomic epidemiology of the emerging pathogen Batrachochytrium dendrobatidis from native and invasive amphibian species in Chile
}

\begin{tabular}{|r|l|}
\hline Journal: & Transboundary and Emerging Diseases \\
\hline Manuscript ID & TBED-RC-242-17.R1 \\
\hline Manuscript Type: & Rapid Communication \\
\hline Date Submitted by the Author: & 13 -Oct-2017 \\
\hline Complete List of Authors: & $\begin{array}{l}\text { Valenzuela-Sánchez, Andrés; Universidad Andres Bello, Centro de } \\
\text { Investigación para la Sustentabilidad } \\
\text { O'Hanlon, Simon; Imperial College London, Department of Infectious } \\
\text { Disease Epidemiology } \\
\text { Alvarado-Rybak, Mario; Universidad Andres Bello, Centro de Investigación } \\
\text { para la Sustentabilidad } \\
\text { Uribe-Rivera, David; ONG Ranita de Darwin } \\
\text { Cunningham, Andrew; Zoological Society of London, Institute of Zoology } \\
\text { Fisher, Matthew; Imperial College London, Department of Infectious } \\
\text { Disease Epidemiology } \\
\text { Soto-Azat, Claudio; Universidad Andres Bello, Centro de Investigación para } \\
\text { la Sustentabilidad }\end{array}$ \\
\hline Subject Area: & \begin{tabular}{l} 
Emerging diseases, Wildlife, Conservation \\
\hline
\end{tabular} \\
\hline
\end{tabular}

\section{SCHOLARONE ${ }^{\text {m }}$}

Manuscripts 
1 Rapid Communication

2 Genomic epidemiology of the emerging pathogen Batrachochytrium dendrobatidis

3 from native and invasive amphibian species in Chile

$4 \quad$ Running title: Genomic epidemiology of Batrachochytrium dendrobatidis in Chile

5 Andrés Valenzuela-Sánchez ${ }^{123}$, Simon J. O'Hanlon ${ }^{4}$, Mario Alvarado-Rybak ${ }^{13}$, David E.

6 Uribe-Rivera $^{2}$, Andrew A. Cunningham ${ }^{3}$, Matthew C. Fisher ${ }^{4}$, Claudio Soto-Azat $^{1}$

$7 \quad{ }^{1}$ Centro de Investigación para la Sustentabilidad \& Doctorado en Medicina de la

8 Conservación, Facultad de Ecología y Recursos Naturales, Universidad Andres Bello,

9 Santiago, Chile.

10 2ONG Ranita de Darwin, Santiago, Chile.

$11{ }^{3}$ Institute of Zoology, Zoological Society of London, Regent's Park, London, UK.

$12{ }^{4}$ Department of Infectious Disease Epidemiology, School of Public Health, St Mary's, 13 Imperial College London, London, UK.

14

15

16

17 Corresponding authors: Andrés Valenzuela-Sánchez, e-mail:

18 andresvalenzuela.zoo@gmail.com; Claudio Soto-Azat, e-mail: csoto@unab.cl 


\section{Summary}

22 Emerging fungal diseases represent a threat to food security, animal and human health 23 worldwide. Amphibian chytridiomycosis, caused by the fungus Batrachochytrium 24 dendrobatidis $(B d)$, has been associated with catastrophic and well-documented 25 amphibian population declines and extinctions. For the first time, Bd was cultured from 26 native and non-native wild amphibians in Chile. Phylogenomic analyses revealed that 27 Chilean isolates AVS2, AVS4 and AVS7 group within the global panzootic lineage of $B d$ 28 (BdGPL) in a single highly supported clade that includes a genotype previously isolated 29 from the United Kingdom. Our results extend the known distribution of BdGPL in South 30 America and suggest a single and relatively recent introduction of $B d G P L$ into the country, 31 providing additional support to the role of anthropogenic activity in the global spread of this 32 panzootic lineage.

33 Keywords: Batrachyla antartandica; Calyptocephalella gayi; chytridiomycosis; emerging 34 infectious disease; fungal disease; Xenopus laevis.

\section{INTRODUCTION}

37 Anthropogenic activity has contributed to the current rise of fungal diseases, representing 38 a threat to food security, animal and human health worldwide (Fisher, Gow, \& Gurr, 2016).

39 Perhaps the most striking example of this problem is chytridiomycosis, an emerging 40 disease of amphibians caused by the fungus Batrachochytrium dendrobatidis (hereafter 41 Bd; Longcore, Pessier, \& Nichols, 1999). Bd occurs in all continents with amphibians, and 42 its impact has been described as 'the most spectacular loss of vertebrate biodiversity due 43 to disease in recorded history' (Skerratt et al., 2007). Initially, this pathogen was 
44 considered to be a rapidly expanding clonal lineage with a single geographical origin 45 (Morehouse et al., 2003). However, further molecular analyses comprising more46 comprehensive methods and a wider geographical representation of isolates, revealed a 47 much more complex evolutionary history, diversity and population dynamics (Farrer et al., 48 2011; Rosenblum et al., 2013). A hypervirulent lineage of $B d$, termed the global panzootic 49 lineage $(B d G P L)$, is widely distributed across the globe and has been associated with 50 catastrophic and well-documented amphibian population declines and extinctions (Farrer 51 et al., 2011; Rosenblum et al., 2013). In contrast, increasing numbers of endemic 52 genotypes have been isolated from amphibians inhabiting Asia, Switzerland, South Africa 53 and Brazil (Farrer et al., 2011; Schloegel et al., 2012; Bataille et al., 2013). To date, none 54 of these have been convincingly linked to host population declines.

In Latin America, $B d$ is a recognized threat to many amphibian species (Lips et al., 56 2006; Soto-Azat et al., 2013). Despite this, there is a knowledge gap on the molecular 57 diversity of $B d$ genotypes occurring in most areas within this region. For instance in Chile, $58 B d$ is widespread, infecting native and invasive anurans, but no attempt has been 59 conducted so far to isolate the fungus or to conduct comparative molecular analyses 60 (Soto-Azat et al., 2013, 2016). This limits our capacity to adequately understand the 61 emergence and epidemiology of chytridiomycosis in the region. The aims of this study are 62 to: 1) describe the isolation and whole-genome sequencing of three $B d$ isolates collected 63 from the skin of wild amphibians inhabiting distinct geographic areas of Chile, and 2) 64 compare these genotypes with a global panel of $B d$. 
67 This study was carried out in accordance with the Bioethics Committee of the Universidad 68 Andres Bello ( $\left.\mathrm{N}^{\circ} 13 / 2015\right)$, the Zoological Society of London's Ethics Committee 69 (WLE709), and the Chilean law (Agriculture and Livestock Service permit N 351/2015). In February 2015, we captured 20 tadpoles of the native marbled wood frog

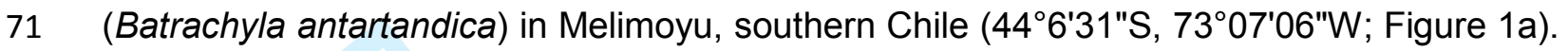
72 In May 2015, we captured 10 wild adults of the invasive African clawed frog (Xenopus

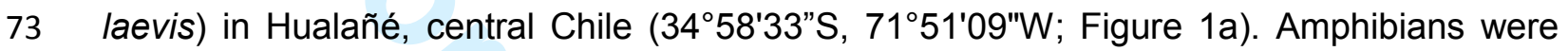
74 kept for no longer than one month, with individuals of each species maintained in each of 75 two separated groups. In order to increase the efficiency of the $B d$ isolation process, 76 animals were first confirmed to be infected using a specific real-time PCR assay (Soto77 Azat et al., 2013). Infected animals were then euthanized under licence by immersion in a 78 diluted solution of tricaine methanesulfonate $(10 \mathrm{~g} / \mathrm{L})$ for one hour. Once death was 79 confirmed, keratinized tissues of $B d$-positive animals (i.e. the whole mouthparts of 80 tadpoles and skin sections of ventral hindlimbs and interdigital membranes of adults) were 81 removed and cultured in a fungal growth medium following the protocol developed by 82 Joyce E. Longcore (https://umaine.edu/chytrids/batrachochytrium-dendrobatidis/directions83 for-isolation). Additionally, in February 2016, during an extensive survey of $B d$ infection in 84 wild amphibians across Chile, we captured one lethargic larvae of the native Chilean frog 85 (Calyptocephalella gayi) in Valdivia (3948'36"S, 7315'21"W; Figure 1a). This individual 86 died in the field during the sampling procedure and exhibited partial depigmentation of the 87 mouthpart, a finding consistent with $B d$ infection. Therefore, we immediately transported 88 the carcass (in refrigerated conditions) to the laboratory; culture of $B d$ was performed as 89 described above. Cultures were grown at $21^{\circ} \mathrm{C}$ in a temperature controlled chamber and 90 were checked every day for zoosporangia development using an inverted light 91 microscope. We confirmed successful isolation (i.e. presence of free-swimming zoospores 
92 and spherical bodies morphologically typical of $B d$ zoosporangia) within 2 weeks. Isolates 93 were passaged no more than three times in order to lessen the chance of genomic change 94 due to prolonged laboratory culture (Voyles et al., 2014) and were subsequently cryo95 archived at $-80^{\circ} \mathrm{C}$ following Boyle et al. (2003).

We performed DNA extraction using the MasterPure ${ }^{\mathrm{TM}}$ Yeast DNA Purification Kit 97 (Epicentre, Wisconsin, USA). DNA extractions were quantified using a Tapestation 2200 98 (Agilent Technologies, California, USA) and Qubit 2.0 fluorimeter (Thermo Fisher 99 Scientific, Massachusetts, USA). We prepared DNA samples for sequencing on an 100 Illumina HiSeq 2000 (Illumina, California, USA). TruSeq Nano 350 gel-free sequencing 101 libraries were prepared for $125+125 \mathrm{bp}$ paired-end sequencing using the Illumina HiSeq 102 high output V4 chemistry.

Sequencing reads were first cleaned of adapter sequences and quality trimmed using 104 cutadapt v1.10 (Martin, 2011). We mapped the reads to the JEL423 reference genome 105 (GenBank assembly accession: GCA_000149865.1) using Burrows-Wheeler Aligner 106 (BWA) v0.7.8 (Li \& Durbin, 2009). We processed the resulting sequence alignment/map 107 (SAM) files using SAMtools v1.3.1 with the 'fixmate' and 'sort' programs to ready the files 108 for variant discovery. We performed the variant discovery in a two-step process using 109 freebayes version dbb6160 (Garrison \& Marth, 2012). In the first step, sorted BAM files for 110 each of the isolates in the phylogeny were independently called to find variant positions. 111 The set of all variant position identified across all samples were merged into a single 112 variant call format (VCF) file. In the second step, each of the samples were re-called using 113 the positions in the merged VCF to produce a squared-off call set (a genotype call is made 114 at every locus for each isolate, including for missing data). All squared-off sample VCF 115 files were processed by vcflib (Garrison, 2016) to break complex variants into allelic 116 primitives and vt (Tan, Abecasis, \& Kang, 2015) to normalize short insertion and deletion 
117 sequences (indels). We quality filtered the VCFs with bcftools version 1.3.1 (Li et al., 2009) 118 to accept only variants with sufficient supporting evidence: sites covered by 4 reads or less 119 were set to missing; potentially polymorphic sites were additionally filtered using the 120 settings recommended in the bcbio.variation.recall squaring-off pipeline (Chapman, 2016), 121 with sites not passing these filters set to homozygous reference, i.e. though there were 122 sequencing reads covering that site, there was not enough evidence to call a variant at 123 that position. We merged the processed VCF files into a single multi-sample VCF and 124 extracted a fasta file of the SNP variant calls. We conducted the phylogenetic analyses 125 using RAxML v8.2.9 with the GTRCAT model with 500 bootstrap runs. The raw read 126 sequences of AVS2, AVS4 and AVS7 are available at the NCBI Sequence Read Archive: 127 https://www.ncbi.nlm.nih.gov/sra under accession numbers $\mathrm{XXX}, \mathrm{XXX}$ and $\mathrm{XXX}$, 128 respectively.

\section{RESULTS AND DISCUSSION}

131 We obtained three isolates across central and southern Chile (Figure 1): AVS2 from $B$. 132 antartandica from Melimoyu, AVS4 from $X$. laevis from Hualañé, and AVS7 from C. gayi 133 from Valdivia. Phylogenomic analyses showed that all Chilean isolates group within 134 BdGPL in a single, highly supported clade (100\% bootstrap support; Figure 1b). The 135 Chilean $B d$ isolates were fixed at $99.4 \%$ of the segregating sites in $B d G P L$ after filtering for 136 missing positions. There were only 2,257 variable sites exclusive to the Chilean $B d$ 137 isolates. Although we compared the genomes of AVS2, AVS4 and AVS7 with an extensive 138 global panel of $B d$, they were shown to be highly divergent from the only known regional 139 endemic lineage (BdBrazil), but clustered with UKTvB, a $B d$ isolate collected from a 140 smooth newt (Lissotriton vulgaris) in 2009 in Kent, United Kingdom (bootstrap support 
$141 \quad 100 \%$, Figure 1b). Inferring phylogenetic relationships within the BdGPL lineage is difficult 142 due to potential SNP homoplasy caused by independent loss of heterozygosity (LOH) 143 events (Rosenblum et al., 2013). We tested the topology produced using SNPs from 144 across the whole autosomal genome against a $1.66 \mathrm{Mb}$ region on supercontig_1.2, which 145 displays a characteristic large-scale loss of heterozygosity ( $\mathrm{LOH}$ ) event, present in all 146 known sequenced isolates of BdGPL (Rosenblum et al., 2013). The topology derived from 147 SNPs in this region (consisting of 19,913 segregating sites), recapitulated the deeply 148 diverged lineages also found in the whole genome tree, with a very flat comb-like structure 149 in the BdGPL lineage due to a lack of SNPs in this region (Figure 2). All 3 Chilean isolates 150 in this study formed a clade (albeit with very little bootstrap support) with isolates from 151 Valencia (VA02), Switzerland (AoCH15), the UK (UKTvB), France (0711.1) and Canada 152 (JEL261), suggesting the clustering with the UK isolate UKTVB in the whole genome 153 analysis may not be artefact. Finally, we used the Weir and Cockerham's estimator in 154 vcftools to perform a sliding-window comparison of $F_{\mathrm{ST}}$ of the Chilean $B d$ isolates against 155 all the other BdGPL isolates. In this analysis, we used a $10 \mathrm{~Kb}$ window, with a $5 \mathrm{~kb}$ step156 size along the genome to identify if any stretches of the Chilean $B d$ population were highly 157 differentiated compared to the rest of the BdGPL clade (Figure 3). We identified several 158 stretches of genome where the $F_{S T}$ estimator was more than two standard deviations 159 greater than the mean of all $F_{S T}$ values, indicating differentiation due to positive selection 160 or reduced rates of recombination may be important at these loci in Chile. Further work is 161 required to determine if any classes of gene are functionally enriched in these high 162 divergence regions.

163 The low number of segregating sites exclusive to the Chilean $B d$ isolates, compared 164 to the total number of sites where the BdGPL isolates are polymorphic, suggest a single 165 and recent instance of long-distance dispersal of $B d$ into Chile, possibly through the 
166 international movement of amphibians, aquatic animals, plants or fomites (Farrer et al., 167 2011; Schloegel et al., 2012). Molecular characterization of further isolates from Chile and 168 neighbouring countries, along with the calibration of a genome-wide molecular clock, is 169 required to confirm this hypothesis. The existence of a unique, recently introduced lineage 170 of $B d$ in Chile would be in contrast with the known history of this pathogen in the near 171 Brazil, where both BdGPL and BdBrazil co-exist, with evidence of multiple hybridization 172 events between them (Jenkinson et al., 2016). This highlights the importance of 173 biosecurity measures to prevent the introduction and establishment of further $B d$ lineages 174 into Chile, as this pathogen has the capability to increase its genomic diversity through the 175 exchange of haplotypes among lineages.

Xenopus laevis is considered as an effective vector capable of disseminating $B d$ into 177 new regions (Soto-Azat et al., 2016) and thus represents a possible route of introduction of 178 BdGPL into Chile. The earliest evidence of $B d$ in Chile is from a four-eyed frog 179 (Pleurodema thaul) individual collected from Concepción in 1970 (Soto-Azat et al. 2013). 180 Although, underestimation of $B d$ is a known problem when examining fixed archived 181 specimens (further discussed in Soto-Azat et al. 2009), hundreds of PCR analyses from 182 archived amphibians have failed to detect $B d$ infections prior to that date in Chile (Soto183 Azat et al., 2013). The exact time of introduction of $X$. laevis into Chile is unknown, but it is 184 thought to have occurred sometime in the 1970s (Jaksic, 1998). Additional means of $185 B d G P L$ introduction, possibly assisted via tourism, research or salmon farming (Liew et al., 186 2017), should also be considered.

The Chilean $B d$ isolates grouped together in our phylogenomic whole-genome 188 analysis with a genotype isolated in 2009 from the United Kingdom (UKTvB). A similar 189 phylogenetic relationship was observed when restricting the analysis to a subset of the 190 genome spanning an LOH event shared by all BdGPL isolates, with isolates from other 
191 European countries and a Canadian isolate also grouping with the Chilean isolates. While 192 there has been no report of mortality caused by the UKTVB, VA02, JEL261 or AoCH15_B 193 isolates, the 0711.1 isolate from the French Pyrenees was associated with amphibian 194 mortalities. In general, BdGPL has been associated with catastrophic mass mortalities and 195 population extirpations in multiple continents (Vredenburg, Knapp, Tunstall, \& Briggs, 196 2010; Farrer et al., 2011; Rosenblum et al., 2013). In Chile, during the last four decades, 197 an extensive extirpation of local populations of the Darwin's frogs (Rhinoderma darwinii 198 and $R$. rufum) has occurred across central and southern Chile. Retrospective, cross199 sectional and capture-recapture data suggest that, among other factors, chytridiomycosis 200 has contributed to the occurrence of these extirpation events (Soto-Azat et al., 2013; 201 Valenzuela-Sánchez et al. 2017). The existence of the hypervirulent BdGPL in Chile 202 provides additional support to this hypothesis. The susceptibility of Rhinoderma spp. to 203 different $B d$ genotypes, however, requires investigation (e.g. controlled infection 204 experiments), because the host-parasite interaction is a complex process that is affected 205 not only by the infectivity and virulence of the parasite but also by host-specific responses 206 and environmental factors (Woodhams et al., 2011).

207 This is the first description of Chilean genotypes of $B d$ and our study gives the first 208 insights into the origin and introduction history of this amphibian pathogen into the country. 209 Our results extend the previously known distribution of BdGPL and provides additional 210 support to the role of amphibian translocations, and perhaps other international 211 movements of aquatic animals, plants or fomites, in the global spread of this panzootic 212 lineage. 
215 We are very grateful to D. Pons, E. Silva, and M. Lima for their helpful comments on an 216 early version of this manuscript. This study was funded by the Dirección General de 217 Investigación y Doctorados, Universidad Andrés Bello, through grants $N^{\circ} \mathrm{DI}-582-14 / \mathrm{l}$ (to 218 A.V.-S.), NDI-53-11/R, DI-7-17/R, and FONDECYT Iniciación N¹1140902 (to C.S.-A.). 219 Genome sequencing was funded by a Natural Environmental Research Council grant to 220 M.C.F.

221

222 Competing interests

223 The authors declare that they have no competing interests.

224

225

\section{REFERENCES}

226

Bataille, A., Fong, J. J., Cha, M., Wogan, G. O., Baek H. J., Lee, H., ... Waldman, B.

227 (2013). Genetic evidence for a high diversity and wide distribution of endemic strains of the pathogenic chytrid fungus Batrachochytrium dendrobatidis in wild Asian amphibians. Molecular Ecology, 22, 4196-4209. doi:10.1111/mec.12385

230

Boyle, D. G., Hyatt, A. D., Daszak, P., Berger, L., Longcore, J. E., Porter, D., ... Olsen, 231 V. (2003). Cryo-archiving of Batrachochytrium dendrobatidis and other chytridiomycetes. Disease of Aquatic Organisms, 56, 59-64. doi:10.3354/dao056059

v0.1.7

Avoid FreeBayes error. Retrieved from https://github.com/chapmanb/bcbio.variation.recall/blob/master/src/bcbio/variation/re call/square (accessed November 12, 2016). 
236 Farrer, R. A., Weinert, L. A., Bielby, J., Garner, T. W., Balloux, F., Clare, F., ... Fisher, 237 M. C. (2011). Multiple emergences of genetically diverse amphibian infecting chytrids 238 include a globalized hypervirulent recombinant lineage. Proceedings of the National 239 Academy of Sciences USA, 108, 18732-18736. doi:10.1073/pnas.1111915108

240 Fisher, M. C., Gow, N. A. R., Gurr, S. J. (2016). Tackling emerging fungal threats to 241 animal health, food security and ecosystem resilience. Philosophical Transactions of 242 the Royal Society B, 371, 20160332. doi:10.1098/rstb.2016.0332

243 Garrison, E., \& Marth, G. (2012). Haplotype-based variant detection from short-read 244 sequencing. arXiv, 1207.3907v2 [q-bio.GN]

245 Garrison, E., (2016). vcflib: A C++ library for parsing and manipulating VCF files. 246 Retrieved from https://github.com/vcflib/vcflib (accessed November 12, 2016).

247 Jaksic, F. M. (1998). Vertebrate invaders and their ecological impacts in Chile. 248 Biodiversity and Conservation, 7, 1427-1445. doi:10.1023/A:1008825802448

249 Jenkinson, T. S., Betancourt Román, C. M., Lambertini, C., Valencia-Aguilar, A., 250 Rodriguez, D., Nunes-de-Almeida, C. H. L., ... James, T. Y. (2016). Amphibian251 killing chytrid in Brazil comprises both locally endemic and globally expanding 252 populations. Molecular Ecology, 25, 2978-2996. doi:10.1111/mec.13599

253 Li, H., \& Durbin, R. (2009). Fast and accurate short read alignment with Burrows254 Wheeler Transform. Bioinformatics, 25, 1754-1760. 255 doi:10.1093/bioinformatics/btp324 
256 Li, H., Handsaker, B., Wysoker, A., Fennell, T., Ruan, J., Homer, N., ... Durbin, R. 257 (2009) The sequence alignment/map (SAM) format and SAMtools. Bioinformatics, $258 \quad 25,1-2$. doi:10.1093/bioinformatics/btp352

259 Liew, N., Mazon, M.J., Wierzbicki, C.J., Hollinshead, M., Dillon, M.J., Thornton, C.R., ... 260 Mostowy, S. (2017). Chytrid fungus infection in zebrafish demonstrates that the 261 pathogen can parasitize non-amphibian vertebrate hosts. Nature Communications, $262 \quad 8,15048$. doi:10.1038/ncomms15048

263 Lips, K.R., Brem, F., Brenes, R., Reeve, J. D., Alford, R. A., Voyles, J., ... Collins, J. P. 264 (2006). Emerging infectious disease and the loss of biodiversity in a Neotropical 265 amphibian community. Proceedings of the National Academy of Sciences USA, 102, 266 3165-3170. doi:10.1073/pnas.0506889103

267 Longcore, J. E., Pessier, A. P., Nichols, D. K. (1999). Batrachochytrium dendrobatidis 268 gen. et sp. nov., a chytrid pathogenic to amphibians. Mycologia, 91, 219-27. 269 doi: $10.2307 / 3761366$

270 Martin, M. (2011). Cutadapt removes adapter sequences from high-throughput 271 sequencing reads. EMBnet.journal, 17, 10-12. doi:10.14806/ej.17.1.200

272 Morehouse, E. A., James, T. Y., Ganley, A. R. D., Vilgalys, R., Berger, L., Murphy, P. 273 J., Longcore, J.E. (2003). Multilocus sequence typing suggests the chytrid pathogen 274 of amphibians is a recently emerged clone. Molecular Ecology, 12, 395-403. 275 doi:10.1046/j.1365-294X.2003.01732.x

276 Rosenblum, E. B., James, T. Y., Zamudio, K. R., Poorten, T. J., Ilut, D., Rodriguez, D., 277 ... Stajich, J.E. (2013). Complex history of the amphibian-killing chytrid fungus 
278

279

280

281

282

283

284

285

286

287

288

289

290

291

292

293

294

295

296

297

298

revealed with genome resequencing data. Proceedings of the National Academy of Sciences USA, 110, 9385-9390. doi:10.1073/pnas.1300130110

Schloegel, L. M., Toledo, L. F., Longcore, J. E., Greenspan, S. E., Vieira, C. A., Lee, M., ... James, T. Y. (2012). Novel, panzootic and hybrid genotypes of amphibian chytridiomycosis associated with the bullfrog trade. Molecular Ecology, 21, 51625177. doi:10.1111/j.1365-294X.2012.05710.x

Skerratt, L. F., Berger, L., Speare, R., Cashins, S., McDonald, K. R., Phillott, A. D., ... Kenyon, N. (2007). Spread of chytridiomycosis has caused the rapid global decline and extinction of frogs. EcoHealth, 4, 125. doi:10.1007/s10393-007-0093-5

Soto-Azat, C., Clarke, B. T., Fisher, M. C., Walker, S. F., Cunningham, A. A. (2009). Non- invasive sampling methods for the detection of Batrachochytrium dendrobatidis in archived amphibians. Diseases of Aquatic Organisms 84, 163-166. doi:10.3354/dao02029

Soto-Azat, C., Valenzuela-Sánchez, A., Clarke, B. T., Busse, K., Ortiz, J. C., Barrientos, C., Cunningham, A. A. (2013). Is chytridiomycosis driving Darwin's frogs to extinction? PLoS One, 8, e79862. doi:10.1371/journal.pone.0079862

Soto-Azat, C., Peñafiel-Ricaurte, A., Price, S. J., Sallaberry-Pincheira, N., García, M. P., Alvarado-Rybak, M., Cunningham, A. A. (2016). Xenopus laevis and emerging amphibian pathogens in Chile. Ecohealth, 13, 775. doi:10.1007/s10393-016-1186-9

Tan, A., Abecasis, G. R., Kang, H. M. (2015). Unified representation of genetic variants. Bioinformatics, 31, 2202-2204. doi:10.1093/bioinformatics/btv112 
299 Valenzuela-Sánchez, A., Schmidt, B.R., Uribe-Rivera, D.E., Costas, F., Cunningham, 300 A.A., Soto-Azat, C. (2017). Cryptic disease-induced mortality may cause host 301 extinction in an apparently stable host-parasite system. Proceedings of the Royal $302 \quad$ Society B 284, 20171176. doi:10.1098/rspb.2017.1176

303 Voyles, J., Johnson, L. R., Briggs, C. J., Cashins, S. D., Alford, R. A., Berger, L., ... 304 Rosenblum, E. B. (2014). Experimental evolution alters the rate and temporal pattern 305 of population growth in Batrachochytrium dendrobatidis, a lethal fungal pathogen of 306 amphibians. Ecology and Evolution, 4, 3633-364. doi:10.1002/ece3.1199

307 Vredenburg, V. T., Knapp, R. A., Tunstall, T. S., Briggs, C. J. (2010). Dynamics of an 308 emerging disease drive large-scale amphibian population extinctions. Proceedings of 309 the National Academy of Sciences USA, 107, 9689-9694. 310 doi:10.1073/pnas.0914111107

311 Woodhams, D. C., Bosch, J., Briggs, C. J., Cashins, S., Davis, L. R., Lauer, A., ... 312 Voyles, J. (2011). Mitigating amphibian disease: strategies to maintain wild 313 populations and control chytridiomycosis. Frontiers in Zoology, 8, 8. 314 doi:10.1186/1742-9994-8-8 


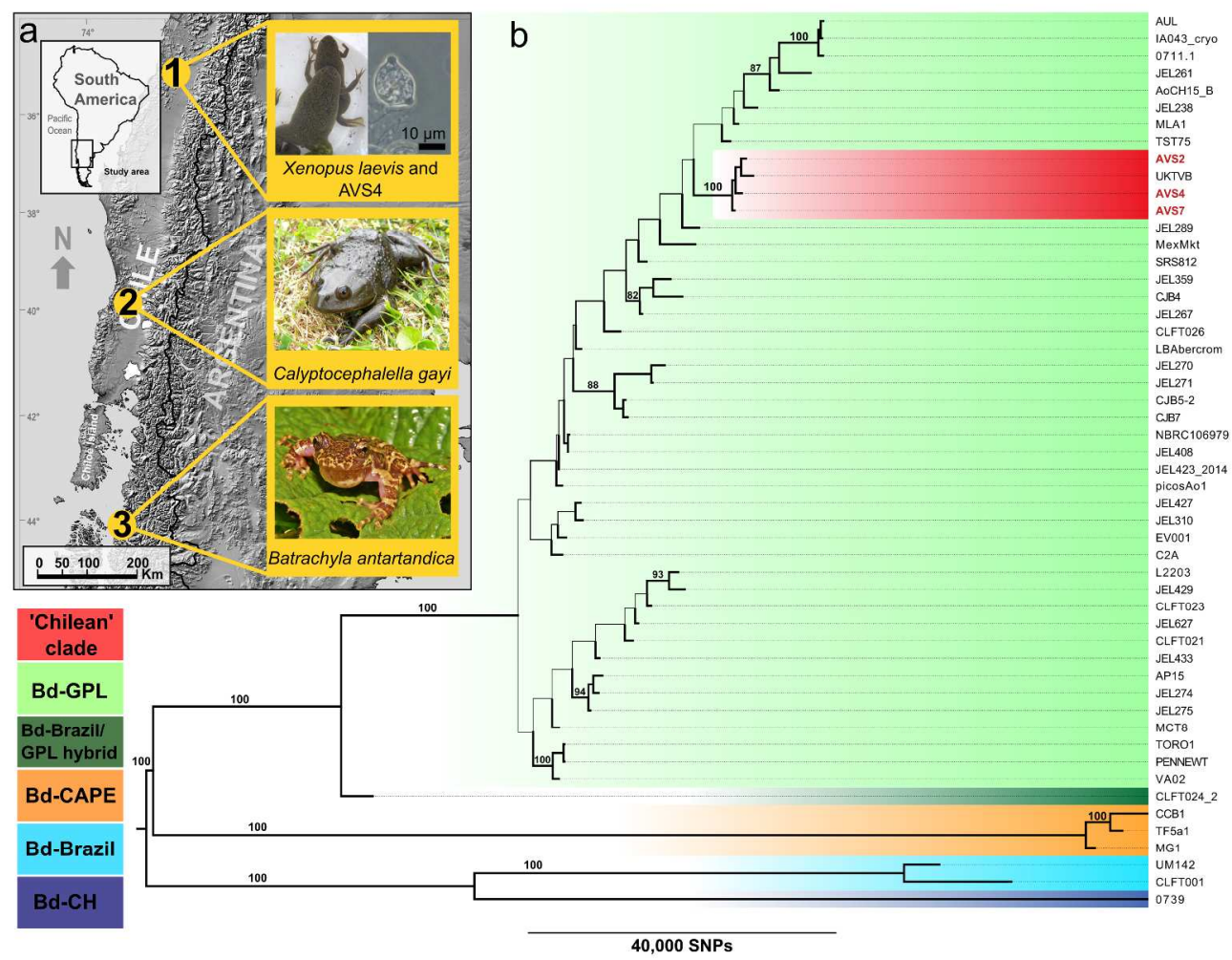

Figure 1. (a) Sites of collection, amphibian species captured and Batrachochytrium dendrobatidis (Bd) [AVS4] isolate obtained from Chile. (b) Global phylogeny of 52 isolates of $B d$ based on 363,497 segregating sites. The clade containing the Chilean $B d$ isolates is highlighted in red, and the Chilean isolates are labelled in red: AVS4 from Xenopus laevis from Hualañé, AVS7 from Calyptocephalella gayi from Valdivia, and AVS2 from Batrachyla antartandica from Melimoyu. The branches of the tree are weighted (thickness) by bootstrap support (500 replicates), with branches with $80 \%$ of support and above labelled. 


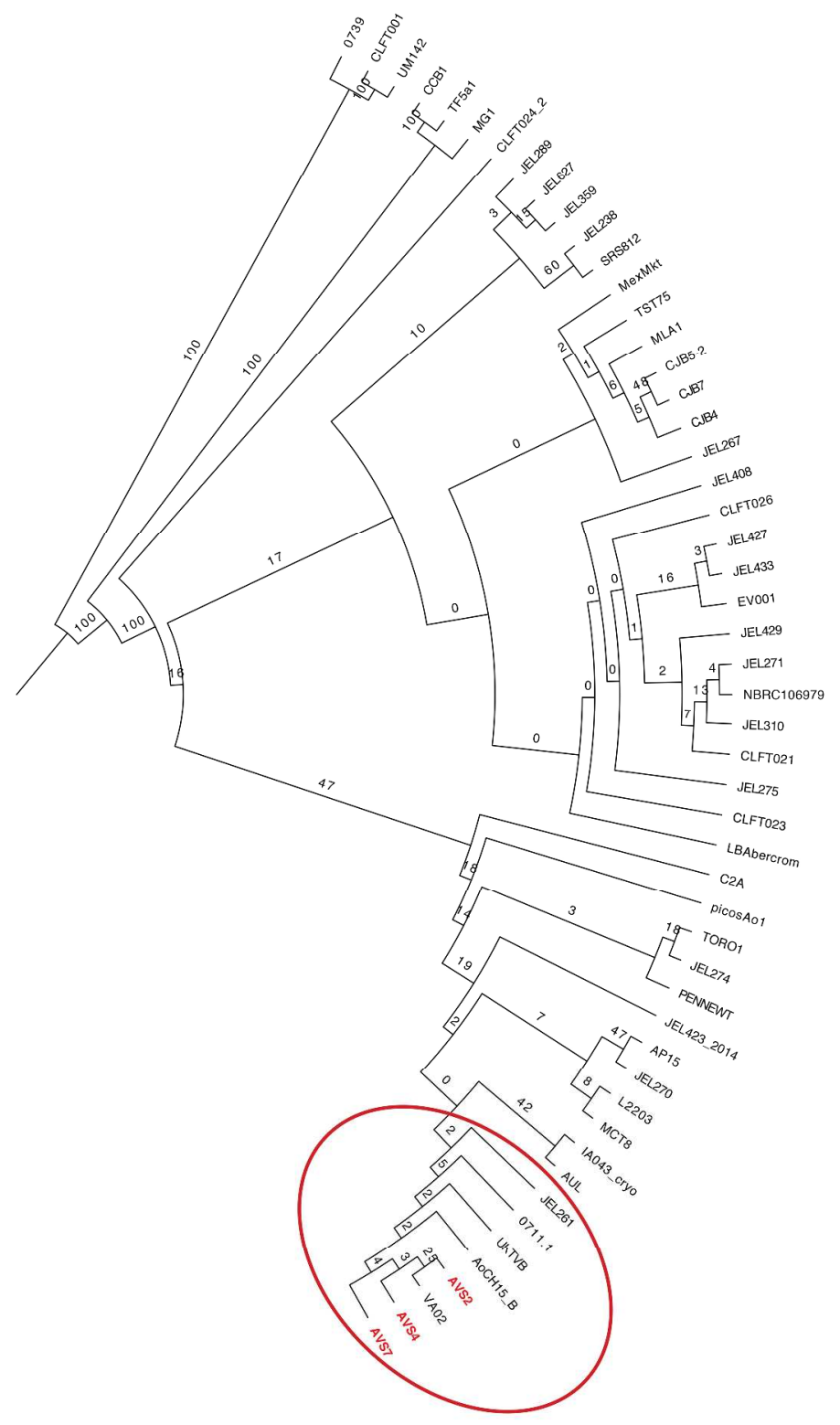

Figure 2. A phylogenetic tree of $B d$ isolates with proportional branch lengths, based on a $1.66 \mathrm{Mb}$ region of supercontig_1.2. This region includes a known loss of heterozygosity (LOH) event shared by all know BdGPL isolates. The Chilean isolates (labelled in red) form a clade (circled in red) with several isolates from Europe (0711.1, AoCH15, UKTVB and VA02), and one from Canada (JEL261). The branch labels indicate bootstrap support from 500 bootstrap replicates.

$251 \times 438 \mathrm{~mm}(300 \times 300 \mathrm{DPI})$ 
Figure 3. Sliding window analysis of population differentiation of Chilean $B d$ isolates against other $B d G P L$ isolates using Weir and Cockerham's F $\mathrm{F}_{\mathrm{ST}}$ estimator. Each point represents a $10 \mathrm{~Kb}$ genomic window, with a $5 \mathrm{~kb}$ step-size. The dashed black line represents the mean $\mathrm{F}_{\mathrm{ST}}(0.0679)$. The solid black line represents the $95 \%$ quantile threshold of the $\mathrm{F}_{\mathrm{ST}}$ estimator $(0.3141)$. Each point is sized and coloured on a log-scale by the number of variants in each window. The legend indicates the colour scale (the number of SNPs included in each window varied from 1 to 177 , with a median of 24). Point size from small to large is scaled from low to high numbers of variants.

$3007 \times 751 \mathrm{~mm}(72 \times 72 \mathrm{DPI})$ 